\title{
Observation of efficient transfer from Mott-Wannier to Frenkel excitons in a hybrid semiconductor quantum dot/polymer composite at room temperature
}

\author{
Sedat Nizamoglu, ${ }^{\text {a) }}$ Xiao Wei Sun, and Hilmi Volkan Demir ${ }^{\text {b) }}$ \\ Department of Electrical and Electronics Engineering, Department of Physics, UNAM- Institute of Material \\ Science and Nanotechnology, Bilkent University, Ankara 06800, Turkey; School of Electrical \\ and Electronics Engineering and School of Physical and Mathematical Sciences, Nanyang Technological \\ University, Singapore 639798
}

(Received 11 October 2010; accepted 22 November 2010; published online 29 December 2010)

\begin{abstract}
Efficient conversion from Mott-Wannier to Frenkel excitons is observed at room temperature. The time-resolved photoluminescence shows that the energy transfer rate and efficiency reach $0.262 \mathrm{~ns}^{-1}$ and $80.9 \%$, respectively. The energy transfer is enabled by strong dipole-dipole coupling in a hybrid inorganic/organic system of $\mathrm{CdSe} / \mathrm{ZnS}$ core/shell heteronanocrystal and poly[2-methoxy-5-(3,7-dimethyl-octyloxy)-1,4-phenylenevinylene] homopolymer composite, and the measured energy transfer efficiencies are consistent with the analytical model. () 2010 American Institute of Physics. [doi:10.1063/1.3529450]
\end{abstract}

The Mott-Wannier and Frenkel excitons are the fundamental Coulomb-correlated light-generation tools in dielectric medium. The interactions of Mott-Wannier and Frenkel excitons provide opportunities in inorganic/organic composite devices. ${ }^{1,2}$ These interactions can be achieved either in a coherent or incoherent way. ${ }^{3}$ For coherent coupling, the electron and hole wave functions in separate systems should strongly interact. However, this condition requires a high degree of structural order. Alternatively, the other interaction channel is the incoherent coupling of Mott-Wannier and Frenkel excitons via Förster-type nonradiative energy transfer (NRET). Such NRET between organic/inorganic hybrids leads to a nonradiative conversion of Mott-Wannier excitons to Frenkel excitons. In a recent study, Blumstengel et al. ${ }^{1}$ showed this kind of exciton conversion process. However, this process was observed only up to a maximum temperature of $100 \mathrm{~K}$ with a moderate energy transfer efficiency level of $50 \%$; the energy transfer was terminated above $100 \mathrm{~K}$ because the excitons were reported to dissociate. To make an efficient system, though, this migration should have a high energy transfer efficiency of $>50 \%$ preferably at room temperature for potential optoelectronic applications. But, efficient exciton conversion at room temperature has not been shown to date. To address this issue, in this letter, we investigate and demonstrate a highly efficient conversion from Mott-Wannier to Frenkel excitons at room temperature. This opens up possibilities for high-efficiency hybrid inorganic/organic devices.

To compose a hybrid organic/inorganic system with high NRET efficiency, NCs and polymers are favorable candidates with their advantageous properties. ${ }^{4-14}$ For this, we choose cyan-emitting $\mathrm{CdSe} / \mathrm{ZnS}$ core/shell heteronanocrystal with Mott-Wannier excitons serving as the donor and orangeemitting poly(2-methoxy-5-(3,7-dimethyl-octyloxy)-1,4phenylenevinylene) (MDMO-PPV) homopolymer with Frenkel excitons serving as the acceptor. Here it is worth

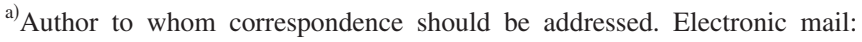
sedatn@ee.bilkent.edu.tr.

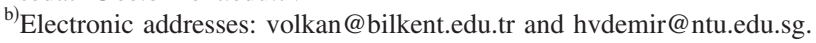

mentioning that a true Mott-Wannier exciton refers to a hydrogen-like bound state intrinsically with its Bohr radius exceeding the crystal lattice constant; however, in our NCs investigated in this work, such a hydrogen-like bound state cannot truly exist because the exciton Bohr radius is comparable to the nanocrystal radius. Nevertheless, the bound electron-hole pair state in these quantum dots is characterized by strong Coulomb interaction, and the notion of exciton can be applied. The character of this Mott-Wannier-like exciton is still fundamentally different than that of a Frenkel exciton. This was previously discussed at length by Gaponenko. ${ }^{15,16}$ Throughout this paper, with the term of Mott-Wannier exciton, we refer to Mott-Wannier-like exciton in a quantum dot. The emission peak of the heteronanocrystals overlaps the absorption peak of the homopolymer beneficial for efficient exciton migration (shown in Fig. S1 in the supplementary information). ${ }^{17}$ We prepared thin films of hybrid inorganic/organic blends by spin-coating at $2000 \mathrm{rpm}$ on quartz substrates (with an acceleration of ca. $700 \mathrm{rpm} / \mathrm{s}$ ). The homopolymer concentration was controlled with great care; the polymer concentrations were approximately 1.5 $\times 10^{17}, \quad 2.9 \times 10^{17}, 4.4 \times 10^{17}$, and $5.3 \times 10^{17} \mathrm{~cm}^{-3}$ in samples $1,2,3$, and 4 , respectively, and the heteronanocrystal concentration in all the samples was around $10^{18} \mathrm{~cm}^{-3}$.

To study the exciton migration, we utilized timeresolved spectroscopy described in Ref 5, with a spectrometer which has an instrument response function (IRF) shown in the inset of Fig. 1. It is worth mentioning that the homopolymer also emits at around $495 \mathrm{~nm}$ (overlapping the NC emission), which could possibly affect the time-resolved behavior, but at the wavelength of $495 \mathrm{~nm}$, the emission of the NCs is much stronger compared to the emission of the homopolymer (see the inset of Fig. 2). So the lifetime of the heteronanocrystals can be clearly distinguished. In the process of fitting, a multiexponential least square error model was adopted, which is convoluted with the IRF, as shown in Eq. (S1) (Ref. 17).

Figure 1 shows the time-resolved spectroscopy of our hybrid homopolymer-heteronanocrystal composites along with that of NC-only solids on quartz substrates at the nano- 


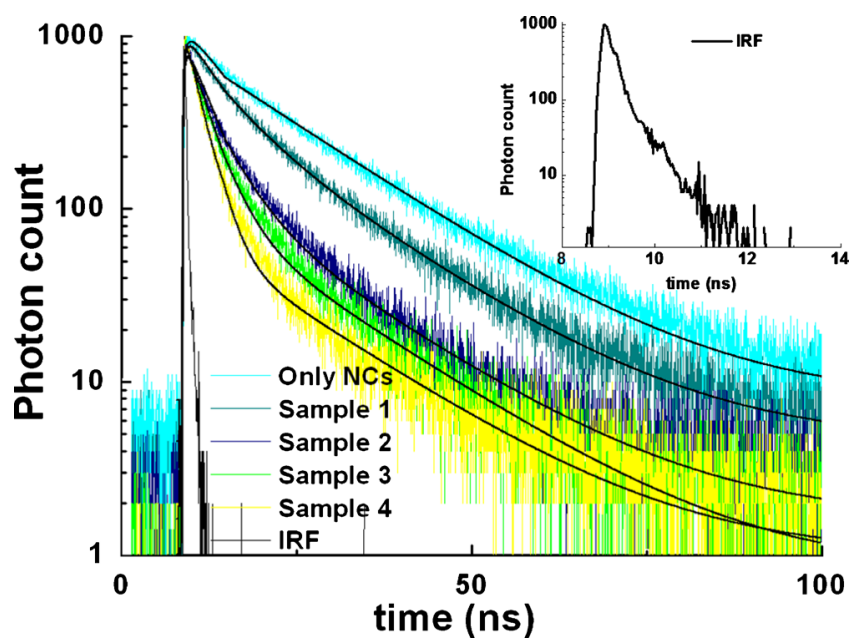

FIG. 1. (Color online) Time-resolved spectroscopy measurement at the peak heteronanocrystal emission wavelength $(495 \mathrm{~nm})$ for the hybrid composite system consisting of heteronanocrystals and homopolymers together (samples 1-4), only nanocrystals on quartz substrate (only NCs), and IRF of the laser diode at $375 \mathrm{~nm}$ using a TCSPC system of PicoHarp 300 with a time resolution of $32 \mathrm{ps}$ at room temperature. The black lines are the fits as described in the text.

crystal donor emission (495 nm). For the NC-only sample, we fit the photoluminescence decay using Eq. (S2) (Ref. 17) with a single lifetime component of $16.16 \mathrm{~ns}$ at room temperature (Table SI), ${ }^{17}$ which is the general lifetime of nanocrystals at room temperature (around tens of nanoseconds). ${ }^{16}$ This serves as the reference decay to distinguish the migration of excitons in the hybrid systems, which corresponds to a decay rate of $0.061 \mathrm{~ns}^{-1}$ (i.e., $\Gamma_{\mathrm{NC}}=0.061 \mathrm{~ns}^{-1}$ ). In sample 1, when the NCs are blended with MDMO-PPV homopolymers, the photoluminescence of NCs starts to quench because of the exciton migration from NCs to polymers. Thus, in addition to the interband recombination process, an extra decay component begins to be observed, as shown in Eq. (1), as a result of the nonradiative energy transfer in the hybrid inorganic-organic sample, where $A_{i}, i=1,2$, are the fitting amplitudes for lifetime components of $\tau_{\mathrm{NC}}$ and $\tau_{\mathrm{NRET}}$.

$$
\begin{aligned}
I_{\text {sample }}(t)= & \int_{-\infty}^{t} \operatorname{IRF}\left(t^{\prime}\right)\left\{A_{1} e^{-\left(t-t^{\prime}\right) / \tau_{\mathrm{NC}}}\right. \\
& \left.+A_{2} e^{-\left(1 / \tau_{\mathrm{NC}}+1 / \tau_{\mathrm{NRET}}\right)\left(t-t^{\prime}\right)}\right\} d t^{\prime} .
\end{aligned}
$$

For sample 1, this additional lifetime component (i.e., $\tau_{2}$ ) is $7.00 \mathrm{~ns}$, which is faster than the recombination lifetime of heteronanocrystals alone. This fast decay rate for sample 1 (i.e., $\Gamma_{\text {sample1 }}$ ) becomes $0.142 \mathrm{~ns}^{-1}$ because of the energy transfer from NCs to homopolymers. We thus determine NRET rate as $\Gamma_{\mathrm{NRET}}=\Gamma_{\text {sample } 1}-\Gamma_{\mathrm{NC}}=0.142-0.061$ $=0.081 \mathrm{~ns}^{-1}$. Furthermore, as the acceptor concentration increases in samples $2-4$, the resulting quenching increases as well. Thus, this fast lifetime component compared with the recombination of heteronanocrystals is shifted from 7.00 to $5.87,4.15$, and $3.08 \mathrm{~ns}$ for samples 2,3 , and 4 at room temperature as a result of the increased NRET from heteronanocrystals to homopolymers, respectively. The corresponding decay rates for samples 2 and 3 are 0.170 and $0.241 \mathrm{~ns}^{-1}$ and we extract their NRET rates of 0.108 and $0.179 \mathrm{~ns}^{-1}$, respectively. Finally, for sample 4, the decay rate reaches a level of $0.324 \mathrm{~ns}^{-1}$, achieving a NRET rate of $0.262 \mathrm{~ns}^{-1}$. Furthermore, the relative amplitude $A_{2}$ arising

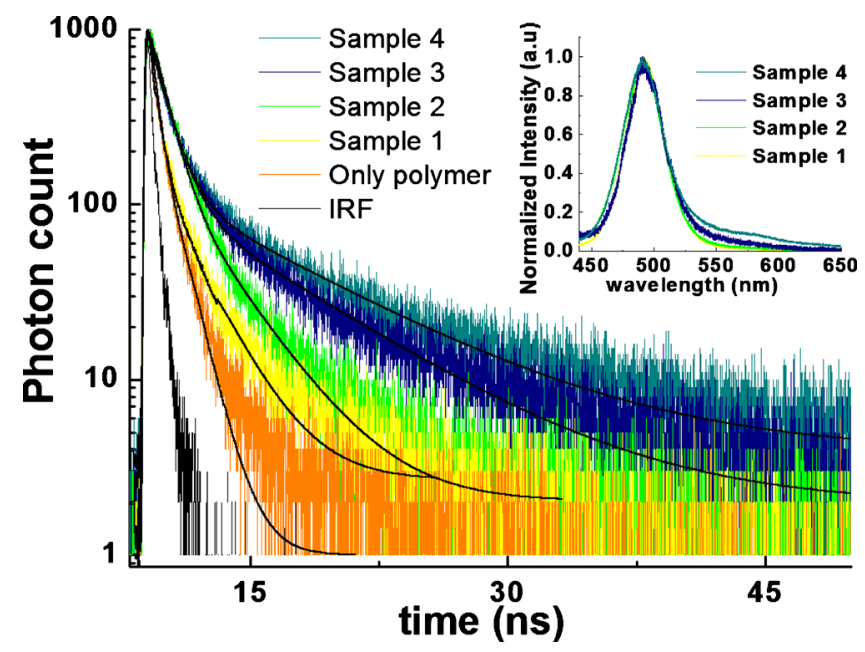

FIG. 2. (Color online) Time-resolved spectroscopy measurement at the peak homopolymer emission wavelength $(585 \mathrm{~nm})$ for the hybrid composite system consisting of heteronanocrystals and homopolymer together (samples 1-4), only homopolymer on quartz substrate, and IRF of the laser diode at $375 \mathrm{~nm}$ using a TCSPC system of PicoHarp 300 with a time resolution of $4 \mathrm{ps}$ at room temperature and the steady-state photoluminescence from samples 1 to 4 is given in the inset. The black lines are the fits as described in the text.

from the energy transfer increases in comparison to that of the recombination component $A_{1}$, showing that the NCs with NRET become more dominant over the NCs without NRET (see Table SI in the supplementary information ${ }^{17}$ ). However, although we observe quenching in nanocrystals here, a possible question is whether the energy is really transferred to polymers or to the surface traps due to the defect states of nanocrystals. Therefore, one also needs to investigate the decay behavior of polymers.

Figure 2 shows the time-resolved spectroscopy of our hybrid heteronanocrystal-homopolymer samples (from sample 1 to sample 4) and the case of only homopolymers at the polymer acceptor emission $(585 \mathrm{~nm})$. In the reference group, the lifetime decay of the MDMO-PPV homopolymers can be represented as biexponentials ${ }^{18}$ with $\tau_{1}=0.228 \mathrm{~ns}$ and $\tau_{2}=1.079 \mathrm{~ns}$ because of their Frenkel-type excitonic behavior. Here the ultrafast recombination of polymers with lifetimes even shorter than $1 \mathrm{~ns}$ is mainly because of the strong electron and hole coupling. This enables the localization of their Frenkel-type exciton in one molecule, in contrast to the case of Mott-Wannier excitons where they can spread (delocalize) over many unit cells of the crystal in the quantum dot, so that their lifetimes are around tens of nanoseconds because of the weaker Coulomb attraction $(<0.1 \mathrm{eV})$. As the acceptor homopolymer concentration increases in the composite, their lifetime increases in contrast to the decreasing lifetime of donor heteronanocrystals.

Moreover, in our hybrid system, this lifetime modification cannot be due to the charge transfer (i.e., Dexter-type transfer) because $\mathrm{ZnS}$ barriers (about three monolayers) in the core/shell heteronanocrystals provide full electronic isolation and prevent tunneling of the electron and hole wave functions. ${ }^{19}$ Furthermore, in the inset of Fig. 2, the steadystate luminescence of the heteronanocrystal-homopolymer composite is shown and the transfer is also visible in the luminescence spectra. From samples 1 to 4 , the emission above the wavelength of $550 \mathrm{~nm}$ coming from the homopolymer is increasing, which is consistent with the de- 


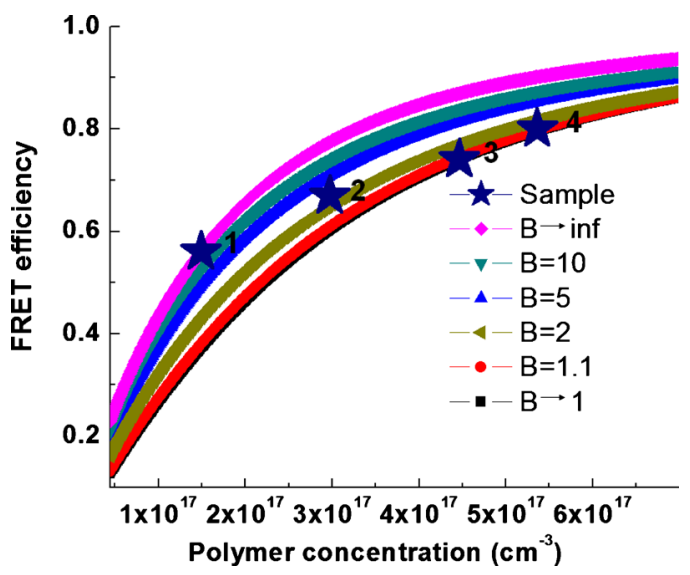

FIG. 3. (Color online) Calculated NRET efficiencies using the analytical model for different $B$ values and measured NRET efficiencies.

creasing donor lifetime and increasing acceptor lifetime. Thus, we can undoubtedly conclude that this is the result of nonradiative Förster-type energy transfer from heteronanocrystals to homopolymers at room temperature.

Here the energy transfer efficiency was calculated as $\eta$ $=\left(\Gamma_{\text {sample }}-\Gamma_{\mathrm{NC}}\right) / \Gamma_{\text {sample }}=\Gamma_{\mathrm{NRET}} / \Gamma_{\text {sample }}{ }^{20}{ }^{20}$ The efficiency for sample 1 corresponds to $56.6 \%$. As we increase the acceptor concentration for samples 2, 3, and 4, the energy transfer efficiencies increase to $63.6 \%, 74.3 \%$, and finally $80.9 \%$, respectively, and for all samples more than half of the excitation energy of heteronanocrystals is transferred to homopolymers at room temperature. Therefore, in our hybrid inorganic/organic structure, the energy is efficiently transformed from the weak Coulomb coupled carriers (MottWannier excitons) to strong Coulomb coupled carriers (Frenkel excitons) at room temperature.

To investigate NRET efficiency $(\eta)$ in our hybrid system, we applied an analysis. In a previous work, Zapunidi et $a l^{21}$ studied quenching of steady-state photoluminescence of donors for varying donor concentrations via NRET. Using both NRET among heteronanocrystals and NRET between heteronanocrystal and homopolymer in our case, we derived the equation for $\eta$ given in Eq. (2).

$$
\begin{aligned}
\eta= & 1-\frac{1}{B^{2}-1}\left(\frac { B ^ { 2 } + ( C q ) ^ { 2 } } { 1 + ( C q ) ^ { 2 } } \operatorname { e x p } \left\{C q \pi\left(\frac{1}{B}-1\right)\right.\right. \\
& \left.\left.+2 C q\left[\arctan (C q)-\frac{\arctan (C q / B)}{B}\right]\right\}-1\right),
\end{aligned}
$$

where $C=4 \pi r_{F}{ }^{3} / 3 \sqrt{Q}, r_{F}$ is the Förster radius for the heteronanocrystal-homopolymer interaction, $q$ is the homopolymer concentration, $Q$ is the quantum efficiency of the heteronanocrystal, and $B$ is the parameter showing the NRET between heteronanocrystals (i.e., if NRET among heteronanocrystals is weak, then $B \rightarrow 1$ and if NRET between heteronanocrystals is strong, then $B \rightarrow \infty$ ). The quantum efficiency of heteronanocrystals is 0.4 and the Förster radius between heteronanocrystal and homopolymer is 9 $\times 10^{-7} \mathrm{~cm}$. Figure 3 shows the calculated and measured NRET efficiencies for different $B$ values, which shows the strength of NRET among NCs. As shown in the figure as the polymer concentration increases, the NRET efficiency be- comes closer to $B \rightarrow 1$ because the intradot energy transfer becomes weaker due to the increased polymer concentration and it can be observed that the experimental results are consistent with the calculated NRET efficiencies, confirming the measured efficiencies and analytical model.

In conclusion, efficient conversion from Mott-Wannier to Frenkel excitons at room temperature has been demonstrated by NRET using a hybrid composite system composed of $\mathrm{CdSe} / \mathrm{ZnS}$ core/shell heteronanocrystals and MDMO-PPV homopolymers. A nonradiative NRET rate of $0.262 \mathrm{~ns}^{-1}$ and an efficiency of $80.9 \%$ were realized. These experimental demonstrations undertaken at room temperature indicate that efficient excitonic interactions of Mott-Wannier and Frenkel excitons are possible in such inorganic/organic hybrids and hold great promise for future hybrid solid state devices.

We acknowledge support from ESF EURYI, EU FP7 Nanophotonics4Energy NoE, TUBITAK EEEAG (107E088, 109E002, 109E004 and 110E010), TUBA-GEBIP and NRF-RF 2009-09.

${ }^{1}$ S. Blumstengel, S. Sadofev, C. Xu, J. Puls, and F. Henneberger, Phys. Rev. Lett. 97, 237401 (2006).

${ }^{2}$ V. M. Agranovich, D. M. Basko, G. C. La Rocca, and F. Bassani, J. Phys.: Condens. Matter 10, 9369 (1998).

${ }^{3}$ S. A. Crooker, J. A. Hollingsworth, S. Tretiak, and V. I. Klimov, Phys. Rev. Lett. 89, 186802 (2002).

${ }^{4}$ D. M. Basko, V. M. Agranovich, F. Bassani, and G. C. La Rocca, Eur. Phys. J. B 13, 653 (2000).

${ }^{5}$ S. Nizamoglu and H. V. Demir, Opt. Express 16, 13961 (2008).

${ }^{6}$ V. I. Klimov, A. Mihkailovsky, S. Xu, A. Malko, J. Hollingsworth, C. Leatherdale, and M. Bawendi, Science 290, 314 (2000).

${ }^{7}$ S. Nizamoglu and H. V. Demir, Opt. Express 16, 3515 (2008).

${ }^{8}$ M. Achermann, M. A. Petruska, S. A. Crooker, and V. I. Klimov, J. Phys. Chem. B 107, 13782 (2003).

${ }^{9}$ T. Franzl, A. Shavel, A. L. Rogach, N. Gaponik, T. A. Klar, A. Eychmüller, and J. Feldmann, Small 1, 392 (2005).

${ }^{10}$ S. V. Gaponenko, V. N. Bogomolov, E. P. Petrov, A. M. Kapitonov, A. Eychmueller, A. L. Rogach, I. I. Kalosha, and U. Woggon, J. Lumin. 87-89, 152 (2000).

${ }^{11}$ S. Nizamoglu, T. Ozel, E. Sari, and H. V. Demir, Nanotechnology 18 065709 (2007).

${ }^{12}$ G. Heliotis, E. Gu, C. Griffin, C. W. Jeon, P. N. Stavrinou, M. D. Dawson, and D. D. C. Bradley, J. Opt. A, Pure Appl. Opt. 8, S445 (2006).

${ }^{13}$ H. Sirringhaus, N. Tessler, and R. H. Friend, Science 280, 1741 (1998).

${ }^{14}$ A. A. Chistyakov, I. L. Martynov, K. E. Mochalov, V. A. Oleinikov, S. V. Sizova, E. A. Ustinovich, and K. V. Zakharchenko, Laser Phys. 16, 1625 (2006).

${ }^{15}$ S. Gaponenko and U. Woggon, Phys. Status Solidi B 189, 286 (1995).

${ }^{16}$ S. V. Gaponenko, Optical Properties of Semiconductor Nanocrystals (Cambridge University Press, Cambridge, England, 1998).

${ }^{17}$ See supplementary material at (http://dx.doi.org/10.1063/1.3529450) for Fig. S1 exhibiting absorption and photoluminescence (PL) spectra of MDMO-PPV homopolymer along with the PL spectrum of CdSe/ZnS core/shell heteronanocrystals and also transmission electron microscopy (TEM) image of the heteronanocrystal and chemical structure of the homopolymer, for Eq. (S1) fitting multiexponential decays, for Eq. (S2) fitting NC-only sample with a single exponential decays, and for Table S1 exhibiting the fitting parameters to the hybrid composite system decays.

${ }^{18}$ M. Anni, L. Manna, R. Cingolani, D. Valerini, A. Cretí, and M. Lomascolo, Appl. Phys. Lett. 85, 4169 (2004).

${ }^{19}$ A. R. Clapp, I. L. Medintz, and H. Mattoussi, ChemPhysChem 7, 47 (2006).

${ }^{20}$ M. Achermann, M. A. Petruska, S. Kos, D. L. Smith, D. D. Koleske, and V. I. Klimov, Nature (London) 429, 642 (2004).

${ }^{21}$ S. A. Zapunidi, Yu V. Krylova, and D. Yu. Paraschuk, Phys. Rev. B 79, 205208 (2009). 